\title{
Editorial
}

\section{Dual chamber single lead pacing}

The idea of single lead atrial synchronous ventricular pacing dates back to the early 1960s when the atrial triggered ventricular paced (VAT) mode was introduced. ${ }^{1}$ Its clinical use was only limited by the fact that an additional atrial lead had to be inserted, a rather difficult procedure with the available technology. However, after the development of specific atrial leads, dual chamber pacing (DDD) also became available and its clinical success made the concept of single lead AV synchronous pacing (VAT, VDD) obsolete.

It took another decade to learn that placement of atrial leads remained difficult, before Antonioli and coworkers re-introduced the concept of a single AV lead in the late 1970s. They observed that atrial depolarisation could reliably be detected through the blood stream by far-field sensing from a unipolar electrode floating in the mid-right atrium. $^{2}$ The most important fact was that no direct wall contact was needed for sensing, which initiated the development of a variety of different leads. After years of experimental and clinical research, the first single AV leads with properly matched VDT pacemakers were implanted in 1980 by the same investigators. ${ }^{3}$ After an initially slow growing clinical experience with unipolar VDD pacemakers, the real breakthrough of VDD pacing took place, when the first bipolar VDD pulse generators with highly sensitive atrial sensing circuits for reliable $P$ wave detection and avoidance of atrial oversensing became available. ${ }^{4-6}$

Today, multiple VDD pacing systems are on the market and their clinical performance is such that usually more than $98 \%$ of all beats are correctly sensed in the atrium and used for triggered ventricular pacing in patients with $\mathrm{AV}$ block. $^{7-10}$ Although the sensed atrial signals from floating electrodes are always smaller and more variable than signals from leads with fixed atrial wall contact, the mean $\mathrm{P}$ wave amplitudes remain very stable over time. In combination with dedicated VDD pulse generators that offer a higher atrial sensitivity than conventional DDD pacemakers, the atrial sensing safety margins typically are 4-6 times higher than the corresponding atrial sensing threshold. With such programming, intermittent atrial undersensing occurs in fewer than $1 \%$ of all atrial depolarisations rendering VDD pacemakers highly reliable in the long term. ${ }^{8} 10$

However, to avoid asynchronous VVI pacing with its associated risks (atrial fibrillation, stroke, heart failure), normal sinus node function is a prerequisite for the long term success of VDD pacing. Therefore, the option of rate adaptation (as offered by virtually all manufacturers) is redundant if patients are correctly selected. Rate adaptive pacing may even be contraindicated in these patients as overriding of sinus rhythm by inappropriate sensor activations may lead to pacemaker syndrome. ${ }^{11}$ Although new onset atrial fibrillation or secondary sinus node dysfunction (binodal disease) may constitute possible indications for rate adaptive pacing, their very low incidence (1-2\%) in published series of VDD paced patients does not justify the routine incorporation of this costly option in VDD pacemakers. ${ }^{5-10}$ Also, the low percentage $(0.2-0.5 \%)$ of VDD paced patients who require upgrading to a DDD system before routine battery replacement seems to support this assumption. ${ }^{5}{ }^{10}$ However, there is little known about the natural deterioration of sinus node function in these patients, and re-evaluation of sinus function is certainly recommended before routine battery replacement of VDD pacemakers.

A specific problem in VDD pacing is the high sensitivity of the atrial sensing circuit, which may cause myopotential or noise sensing leading to inappropriate pacemaker tachycardia (noise tracking). However, with bipolar atrial sensing (diagonal dipoles or closely spaced rings), atrial oversensing has clinically become negligible, although myopotential sensing may still be provoked by forced arm exercise in up to $5 \%$ of patients during laboratory testing. ${ }^{10}$

Given these excellent results and the ease of single lead implantation, for many investigators VDD pacing has become the preferred mode for patients with AV block and normal sinus node function, although there are no data from randomised studies that would prove the relative merits of VDD compared with DDD pacing in this population. Unfortunately, the only ongoing large scale multicentre trial addressing this issue (INSIDE study) is very likely to show no significant differences as it lacks statistical power.

\section{Single lead dual chamber (DDD) pacing}

Although VDD pacing has become a very valuable option in patients with AV block and normal sinus node function, the lack of ability to pace the atrium rules out this system in most patients with sick sinus syndrome. ${ }^{12}$ Therefore, most manufacturers have now focused on studying the possibilities of single lead dual chamber pacing.

Currently there are two different approaches. The first aims at getting good and stable atrial capture from the same or slightly modified bipolar atrial floating dipoles that have been developed for VDD pacing. The major drawback with this technique is the high atrial pacing threshold due to a significant loss of the delivered energy in the blood stream. Typically, atrial thresholds are in the range of $4-8 \mathrm{~V}$ at a pulse width of $0.5 \mathrm{~ms}$ and in $10-25 \%$ of patients no atrial capture can be achieved. ${ }^{13}$ As well as high energy consumption, phrenic nerve stimulation is another problem that makes single lead DDD pacing impossible in up to $30-50 \%$ of patients. ${ }^{13}$ Therefore, more effective pacing techniques such as the OLBI mode (overlapping biphasic stimulation) are currently being investigated. ${ }^{15}$ With the OLBI technique, two sequential monophasic impulses of opposite polarity are delivered for pacing. This has been shown to reduce the atrial capture threshold significantly, whereas the threshold for diaphragmatic stimulation remains unchanged. ${ }^{13}$ In one study of OLBI pacing from bipolar atrial floating electrodes, chronic atrial pacing without diaphragmatic stimulation could be achieved in $76 \%$ of patients at three months of follow up. However, considering that two sequential impulses of twice threshold strength $(2.5 \pm 0.8 \mathrm{~V}$ at $0.5 \mathrm{~ms})$ are required for chronic OLBI stimulation, the energy drain from the battery used for atrial stimulation remains about 3-6 times higher than pacing via conventional atrial leads. ${ }^{14}$ Assuming that about $50-75 \%$ of all atrial beats are paced beats in patients with sick sinus syndrome, this would shorten the longevity of a modern $\operatorname{DDD}(\mathrm{R})$ pulse generator by at least $30-40 \%$. 
The second approach in single lead DDD pacing is targeted at achieving direct atrial wall contact with the use of different fixation mechanisms. So far, some experimental and clinical studies with prototype leads have shown promising results with low and stable atrial capture thresholds. ${ }^{16-18}$ However, the problem of stable lead fixation in the atrium has not yet been solved, and the different modalities currently investigated (hooks, screws, spirals, loops, etc) are rather demanding in terms of surgical handling. This raises the key question of fixed wall contact DDD single leads: if a highly experienced expert is needed to place one of these leads, what is the gain compared to implanting a conventional atrial lead with excellent long term performance?

Therefore, given the current status of knowledge, one may conclude that inserting a VDD pacemaker in a patient with AV block and normal sinus node function has become a simple and reliable solution in (almost) everyone's hands. At this time, however, patients with sick sinus syndrome should still receive a properly inserted atrial lead that guarantees low atrial pacing thresholds and an optimal longevity of the pulse generator.

Cardiac Unit, University Hospital,

S OSSWALD

CH-4031 Basel, Switzerland

1 Nathan DA, Samet P, Center S, et al. Clinical and physiologic studies of a new type implantable synchronous pacer. Progr Cardiovasc Dis 1964;6:538-65.

2 Antonioli GE, Grassi G, Baggioni GF, et al. A simple new method for atria triggered pacemaker. G Ital Cardiol 1980;10:679-89.

3 Antonioli GE, Grassi G, Marzaloni M, et al. A new implantable VDT pacemaker using a single double-electrode cathter. In: GA Feruglio, ed Cardiac pacing (Europacing Florence). Padova: Piccin Medical Books 1982:1093-9.
4 Longo E, Catrini V. Experience and implantation techniques with a new single-lead VDD pacing system. PACE 1990;13:927-36.

5 Antonioli GE, Ansani L, Barbieri D. Italian mulicener study on single lead VDD pacing system using a narrow atrial dipole spacing. PACE 1992;15:1890-3.

6 Lau CP, Tai YT, Li JP, et al. Initial clinical experience with a single pass VDDR pacing system. PACE 1992;15:1894-900.

7 Crick JCP. European multicenter prospective follow-up study of 1002 implants of a single lead VDD pacing system. PACE 1991;14:1742-4.

8 Naegeli B, Osswald S, Pfisterer M, et al. $\operatorname{VDD}(\mathrm{R})$ pacing: short- and long-term stability of atrial sensing with a single lead system. PACE 1996; 19:455-64.

9 Lau CP, Leung SK, Lee IS. Comparative evaluation of acute and long-term clinical performance of two single lead atrial synchronous ventricular (VDD) pacemakers: diagonally arranged versus closely spaced bipolar ring electrodes. PACE 1996;19:1574-81.

10 Osswald S, Cron T, El Allaf D, et al. Clinical performance of the model 425-11, -13, -16 UniPass pacing lead: results of a multicenter international study. In: Vardas PE, ed. Europace'97 Proceedings. Bologna: Monduzzi Editore, 1997:599-602.

11 Osswald S, Leiggener C, Buser $\mathrm{P}$, et al. Benefits and limitations of rate-adaptive pacing under laboratory and daily life conditions in patients with minute-ventilation single-chamber pacemakers. PACE 1996;19:890-8.

12 Andersen HR, Thuesen L, Bagger JP, et al. Prospective randomised trial of atrial versus ventricular pacing in sick-sinus syndrome. Lancet 1994;344: $1523-8$.

13 Tse HF, Lau CP, Leung SK, et al. Single lead DDD system: a comparative evaluation of unipolar, bipolar, and overlapping biphasic stimulation and the effects of right atrial floating electrode location on atrial pacing and sensing thresholds. PACE 1996;19:1758-63.

14 Rey JL, el Ghelbazouri F, Tribouilloy C. Dual chamber pacing with a single lead system: initial clinical results. PACE 1996;19:1777-9.

15 Hartung WM, Hidden-Lucet F, McTeague K, et al. Overlapping biphasic stimulation: a novel pacing mode with low capture thresholds [abstract]. Circulation 1994;90:I-90.

16 Hirschberg J, Ekwall C, Strandberg H, et al. A new dual chamber single lead system. PACE 1994;17:1870-2.

17 Naegeli B, Straumann E, Gerber A, et al. A new approach for $\mathrm{DDD}(\mathrm{R})$ pacing using a single-pass DDD lead. Am f Cardiol. [In press.]

18 Morgan K, Bornzin JF, Wolsleger W, et al. A a new single pass DDD lead [abstract]. PACE 1211;20:1211.

\section{STAMPS IN CARDIOLOGY}

\section{Atherosclerosis and myocardial infarction}

Very few stamps have appeared relating directly to atherosclerosis and myocardial infarction. The Cape Verde 4 escudo stamp was released in 1980 as part of the issue for the World Health Day antismoking campaign of the World Health Organisation. Careful consideration has gone into the design, which shows the three forms of smoking, a large area of myocardial infarction, and the chest leads of the electrocardiogram showing a full thickness anterior infarction. In this respect this is a unique stamp. The Dominican Republic issued a set of three stamps in 1979 to commemorate the Dominican Cardiology Institute. The 3 cent stamp shows an atherosclerotic artery in the foreground and an occluded left anterior descending vessel within the diagrammatic heart in the background.

M K DAVIES A HOLLMAN 